\title{
Mean blastomere diameter may predict clinical pregnancy in long agonist protocol intracytoplasmic sperm injection cycles with single embryo transfer
}

\author{
Suleyman Guven ${ }^{1 *}$, Sebnem Alanya Tosun² and Emine Seda Guvendag Guven ${ }^{1}$
}

\begin{abstract}
Background: The main purpose of this study was to investigate the effect of mean blastomere diameter (MBD) on pregnancy rates in in vitro fertilization (IVF) cases undergoing the long agonist cycle protocol. A total of 84 cases were evaluated within the scope of this observational prospective study. All cases were normoresponders, under 35 years old, with the long agonist protocol applied and single embryo (grade I or II) transfer performed. On the third day after ICSI, each embryo selected for transfer was subjected to measurement of the mean blastomere diameter $(\mathrm{MBD})$ at $\times 25$ magnification.
\end{abstract}

Results: The mean female age was $30.14 \pm 3.32$ years, and the total clinical pregnancy rate was $33.3 \%$. In the group that got pregnant, MBD was found to be statistically significantly higher than in the nonpregnant group. In terms of predicting clinical pregnancy, when the MBD value of $49.73 \mu \mathrm{m}$ was accepted as the best cutoff value, the sensitivity was calculated as $75 \%$ and specificity as $53.6 \%$. Clinical pregnancy rate was $18.9 \%$ in cases below this value, whereas clinical pregnancy rate was $44.7 \%$ in cases with this value and above. In other words, when the MBD value rose above $49.73 \mu \mathrm{m}$ from a value below $49.73 \mu \mathrm{m}$, the clinical pregnancy rate increased by an average of 2.3 times.

Conclusions: With MBD measurement, it is possible to select the embryo with the best implantation capability in microinjection cycles with the long luteal agonist protocol.

Keywords: ART, Clinical pregnancy, Embryo, Mean blastomere diameter

\section{Background}

The intracytoplasmic microinjection process continues to be used as the best option in cases with severe male infertility and in cases where success cannot be achieved in classical in vitro fertilization (IVF) [1]. With this method, compared with the classical IVF method, the stages of attachment to the oocyte membrane by the movement of the sperm, passing through the membrane, and reaching the cytoplasm will be bypassed. According

\footnotetext{
* Correspondence: drsuleymanguven@yahoo.com

'Departments of Obstetrics and Gynecology, Karadeniz Technical University

Faculty of Medicine, Farabi Hospital, 61080 Trabzon, Turkey

Full list of author information is available at the end of the article
}

to current data, eliminating these stages by microinjection does not cause serious problems and congenital anomalies in the baby [2-4].

From another perspective, it is clear that clinical pregnancy rates will increase in the case of double embryo transfer in pregnancies obtained through assisted reproductive techniques. However, with two-embryo transfer, the live birth rate, the rate of bringing a baby home, decreases and the risk of multiple pregnancy increases [5]. These risks may be reduced by a well-selected single embryo transfer.

Over time, embryologists have developed grading systems to form a common language worldwide to describe 
embryo morphology. Currently, well-defined embryo assessment criteria that have been used in IVF clinics consist of morphologic features such as fragmentation, symmetry, multi-nucleation, and vacuolation [6].

In the detailed literature search, no study was found on use of the mean blastomere diameter (MBD) to evaluate the morphological features of the third day embryo in ICSI cycles. Based on our experience and preliminary study results, we hypothesized that the mean blastomere diameter (MBD) would be higher in cycles achieving clinical pregnancy.

Hence, the main purpose of this study was to investigate the effect of MBD on pregnancy rates in IVF cases undergoing the long agonist cycle protocol. This would be an appropriate way to choose the best embryo to maximize the clinical pregnancy rate.

\section{Methods}

A total of 84 cases (86 ICSI cycles) were evaluated within the scope of this observational prospective study. The research period was 14 months, and approval was obtained from the Ethics Committee of Karadeniz Technical University Faculty of Medicine.

All cases were normoresponders, under 35 years old, with the long agonist protocol applied and single embryo (grade I or II [ $<10 \%$ fragmentation] with 8 cells) transfer performed. On the third day after ICSI, each embryo selected for transfer was subjected to measurement of the MBD at $\times 25$ magnification.

The standard luteal long lucrin ovulation induction protocol, details of which were given in our previous study, was applied in all cases [7]. Women with high progesterone $(\geq 0.8 \mathrm{ng} / \mathrm{mL})$ levels on HCG day were excluded.

Egg collection was performed at the 36th $\mathrm{h}$ after HCG, microinjection was performed in all cases, fertilization was evaluated $17 \mathrm{~h}$ after ICSI, and the presence of 2 PN was confirmed in all cases. ICSI was performed approximately $38 \mathrm{~h}$ after HCG triggering. The embryo grading system recommended by Hardarson et al. was used [8]. Day 3 embryo transfer was preferred. Embryo transfer was performed an average of 75 $\mathrm{h}$ after ICSI. All patients had single embryo transfer. Embryo transfer was performed by a single clinician (SG) and embryologist under the guidance of ultrasonography. Soft catheter (Wallace, Smith Co., UK) was used for embryo transfer. Type 1 embryo transfer detailed below was performed in all cases. The cases were prepared for embryo transfer under lithotomy position with a full bladder. A sterile Graves speculum was placed, and the cervix was cleaned with saline solution first, then washed with culture solution. No trial transfer was made. The uterus endometrial cavity was visualized by pelvic ultrasonography. The embryo brought with a soft catheter was gently placed $1-2 \mathrm{~cm}$ below the fundus under the guidance of ultrasonography. The transfer catheter was removed from the cavity by gently turning it. The fundus was not touched during the embryo transfer. The removed catheter was checked under the microscope for embryo retention, blood, and mucus. No blood or mucus was observed. For luteal phase support, all cases were recommended to use vaginal progesterone gel twice a day in the morning and evening, starting from the day of egg collection until the time of pregnancy test.

All cases had normal sperm parameter values, given below. Sperm volume was above $1.5 \mathrm{~mL}$, sperm count was over 15 million/mL, sperm progressive motility value was above $32 \%$, and sperm morphology was over $4 \%$, according to Tygerberg's method [9].

On the third day after ICSI, each embryo selected for transfer was subjected to measurement of the MBD at $\times$ 25 magnification. MBD was measured after an average of $72 \mathrm{~h}$ after ICSI. In all cases, embryos containing only 8 blastomeres were considered within the scope of the study and selected for transfer. Embryos derived from giant oocytes or oocytes with large perivitelline space were not included. Embryos containing more than 20\% asymmetric blastomeres were not included in the study. Only one photo showing all blastomeres was selected for MBD measurement. The following procedure was applied for MBD calculation. First, an embryo containing 8 cells, no more than $10 \%$ fragmentation, and less than $20 \%$ blastomere asymmetry was selected for transfer by the single embryologist. Then, two diameters perpendicular to each blastomere were measured. These two diameters were summed up and divided in half. These measurements were made for every 8 blastomeres. Average values of each blastomere were summed and divided by 8 , and the MBD was found in micrometers (Fig. 1). The mean zona thickness was calculated by the following formula: Zona thickness (micrometers) was measured in two different areas around the embryo, summed up, and then divided by two.

Cases with normoresponder female and normal male evaluation tests were selected according to the study protocol. The same ovarian stimulation protocol was applied. Embryos were evaluated for fertilization and cleavage by a single embryologist (OY, with 10 years of experience in an IVF laboratory). MBD and zona thickness were measured by a single embryologist (OY). This process took an average of $3 \mathrm{~min}$. Day 3 embryo transfer was performed. On the 12th day after the embryo transfer, a pregnancy test was performed in the blood. In cases with a positive pregnancy test, detection of heart rate via transvaginal ultrasound during the follow-up period was accepted as clinical pregnancy. 


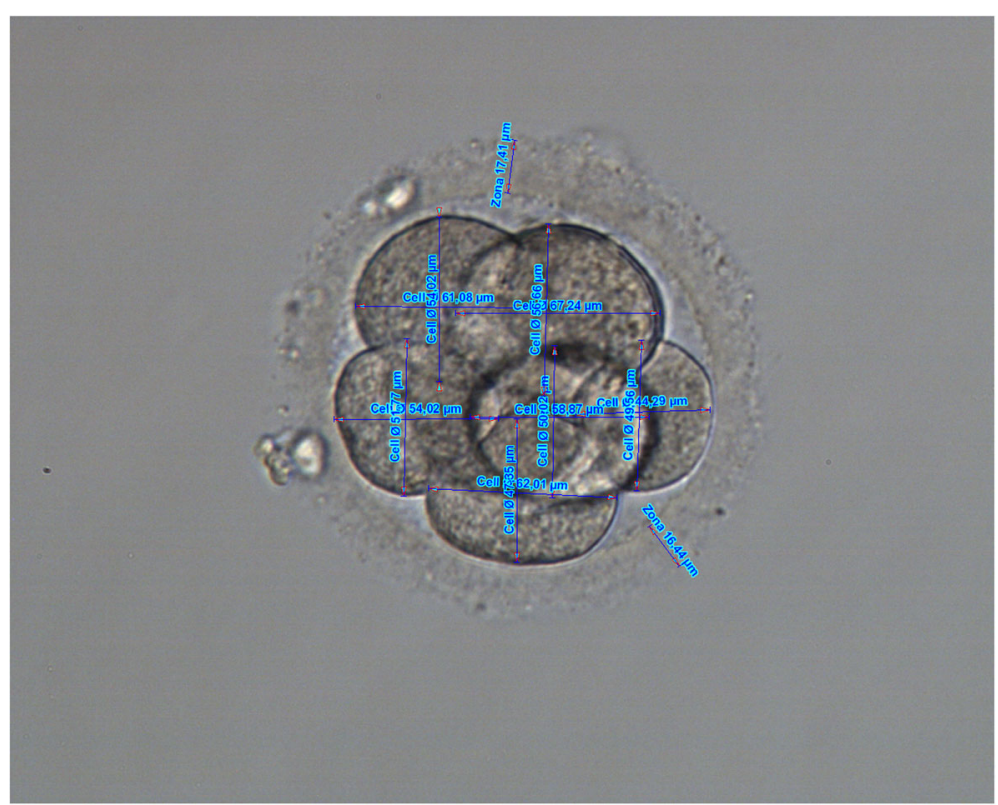

Fig. 1 Photograph of day 3 embryo showing the measurement of zona thickness in the embryo and mean blastomere diameter in each blastomere

Clinical pregnancy was defined as an embryo with a heartbeat detected under ultrasonographic evaluation 4 weeks after embryo transfer. Fisher's exact chi-square test, Student's $t$ test, and ROC analysis were used for statistical analysis. The SPSS computer program was used for statistical analysis. A $p$ value of less than 0.05 was considered statistically significant. According to our preliminary study results, the MBD was accepted as 49 and a standard deviation of 3.5 in the group that could not conceive, and 52 in the group that did conceive. According to these data, when there were at least 21 cases in each group, alpha was 0.05 and power was calculated as 0.8 .

Table 1 Comparison of clinical and embryologic characteristics in pregnant and nonpregnant groups

\begin{tabular}{|c|c|c|c|}
\hline Parameters & Pregnant $(n=28)$ & Nonpregnant $(n=56)$ & $p$ \\
\hline Female age (yr.) & $28.57 \pm 3.26$ & $28.93 \pm 3.38$ & 0.645 \\
\hline Male age (yr.) & $33.32 \pm 4.60$ & $34.68 \pm 5.06$ & 0.236 \\
\hline Infertility duration (yr.) & $5.63 \pm 4.14$ & $6.70 \pm 5.01$ & 0.222 \\
\hline Cause of infertility (\%) & & & $0.117^{\mathrm{a}}$ \\
\hline Female factor & $20(39.2 \%)$ & $31(60.8 \%)$ & \\
\hline Mild male factor & $8(24.2 \%)$ & $25(75.8 \%)$ & \\
\hline Body mass index $\left(\mathrm{kg} / \mathrm{m}^{2}\right)$ & $21.98 \pm 1.22$ & $21.91 \pm 1.48$ & 0.821 \\
\hline Basal FSH (mIU/mL) & $6.07 \pm 1.86$ & $7.40 \pm 4.23$ & 0.195 \\
\hline Basal E2 (pg/mL) & $61.75 \pm 48.123$ & $59.32 \pm 23.25$ & 0.801 \\
\hline Basal antral follicle count (no.) & $12.67 \pm 5.19$ & $11.00 \pm 6.60$ & 0.257 \\
\hline Amount of total FSH used (IU/L) & $1983.33 \pm 305.95$ & $2068.27 \pm 348.11$ & 0.288 \\
\hline Length of ovarian stimulation (day) & $8.81 \pm 1.36$ & $9.17 \pm 1.56$ & 0.315 \\
\hline Endometrial thickness on HCG day (mm.) & $9.28 \pm 0.70$ & $9.12 \pm 0.67$ & 0.306 \\
\hline Mean E2 on HCG day $(\mathrm{pg} / \mathrm{mL})$ & $1643.65 \pm 673.89$ & $1380.34 \pm 594.97$ & 0.071 \\
\hline Number of MII oocyte retrieved & $8.78 \pm 4.32$ & $8.25 \pm 3.55$ & 0.557 \\
\hline Grade I embryo (\%) & $22(78.6 \%)$ & $45(80.4 \%)$ & 0.530 \\
\hline Total fertilization rate (\%) & $64.94 \pm 15.10$ & $54.57 \pm 20.80$ & 0.201 \\
\hline Mean zona thickness $(\mu \mathrm{m})$ & $16.41 \pm 2.68$ & $16.11 \pm 2.33$ & 0.595 \\
\hline Mean blastomer diameter $(\mu \mathrm{m})$ & $52.09 \pm 3.98$ & $49.44 \pm 3.62$ & 0.003 \\
\hline
\end{tabular}

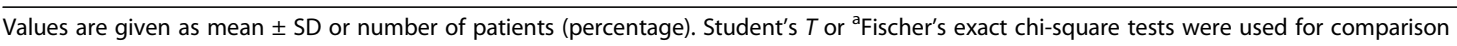




\section{Results}

The mean female age in the study group was $30.14 \pm$ 3.32 years. In all cases, the clinical pregnancy rate was $33.3 \%$, and the rate of taking a baby home was $31.0 \%$.

The comparison of clinical and laboratory/embryologic data is given in Table 1. The MBD was found to be statistically significantly higher in the pregnant group $(n=$ 28) compared with the nonpregnant group $(n=56)$ $(52.09 \pm 3.98$ vs. $49.44 \pm 3.62 \mu \mathrm{m}$, respectively, $p=$ 0.003 , Student's $t$ test).

According to the ROC analysis test result, the area under the curve for MBD was 0.680 (95\% CI $0.562-$ 0.798) (Fig. 2). In terms of predicting clinical pregnancy, when the MBD value of $49.73 \mu \mathrm{m}$ was accepted as the best cutoff value, the sensitivity was calculated as $75 \%$ and specificity as $53.6 \%$. Clinical pregnancy rate was $18.9 \%$ in cases below this value, whereas clinical pregnancy rate was $44.7 \%$ in cases with this value and above ( $p=0.01$, Fisher's exact chi-square test). In other words, when the MBD value rose above $49.73 \mu \mathrm{m}$ from a value below $49.73 \mu \mathrm{m}$, the clinical pregnancy rate increased by an average of 2.3 times.
Similarly, the live birth rate was $23.1 \%$ when the MBD was below 49.73, and the live birth rate was $76.9 \%$ when the MBD was 49.73 and above $(p=0.008$, Fisher's exact chi-square test). The live birth rate increased 3.3 times when the MBD value increased from below 49.73 to 49.73 and above.

\section{Discussion}

The main goal of this study was to determine the embryo with high adherence potential to the endometrium that would increase the clinical pregnancy rate in ICSI cycles by morphological parameter evaluation. The result clearly showed that MBD higher than $49.73 \mu \mathrm{m}$ could predict embryos more ideal for transfer, thus increasing the clinical pregnancy rates in ICSI cycles.

Embryo morphology evaluation on day 3 is useful in the determination of the best embryos [10]. In the past, more than two embryos were transferred to increase pregnancy rates. However, it has been found that this approach also increases the rate of multiple pregnancy and poses additional medical risks for the mother and

\section{ROC Curve}

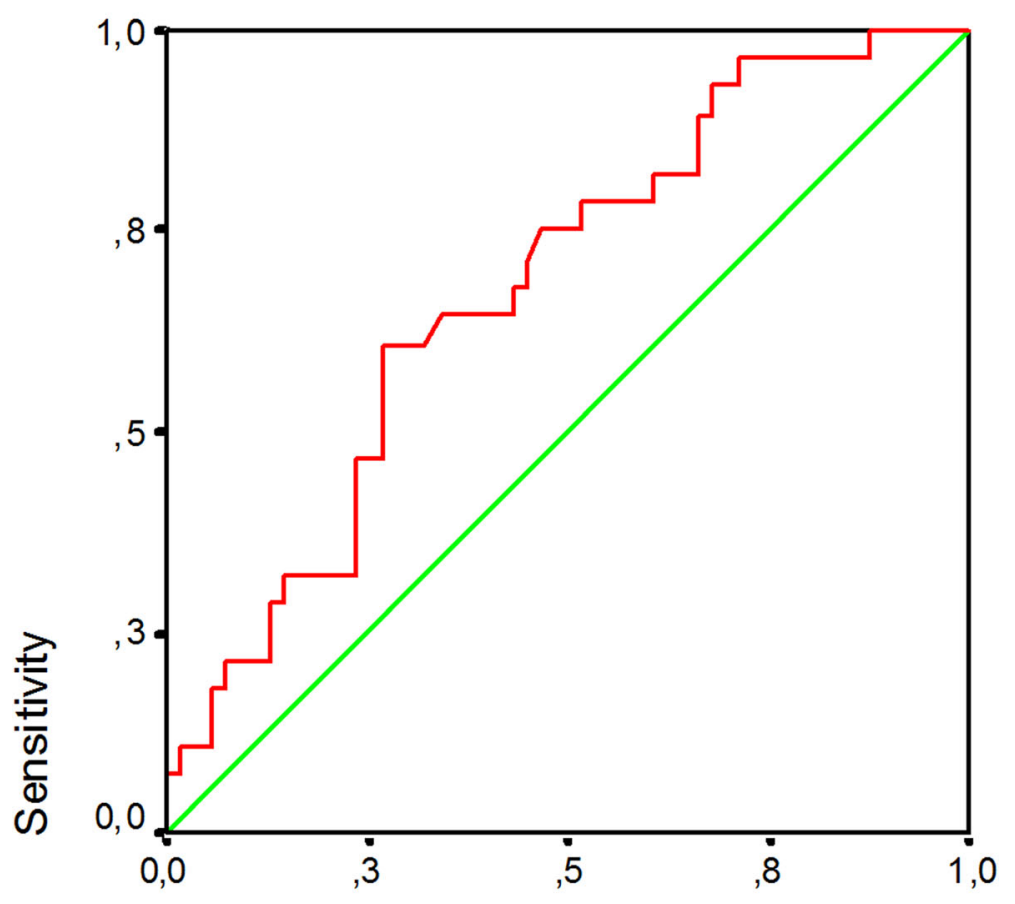

\section{1 - Specificity}

\section{Diag onal segments are produc ed by ties.}

Fig. 2 Receiver operating characteristic curve to calculate the best MBD value for predicting clinical pregnancy 
baby [11]. Changing embryo selection criteria over the years has brought innovations in the selection of the best embryo to implant, and the process of recovery in clinical pregnancies and a decrease in multiple pregnancies has started [11, 12]. The risks that develop with the transfer of excess embryos have been included in the relevant scope of international authorities on ART, and many associations have published guidelines on the subject [13]. All these developments have put pressure on IVF centers to transfer a small number, preferably a single embryo. This has led to the intensification of research on the best embryo selection criteria.

Although many morphological evaluation methods related to blastomeres have been defined, the effect of MBD on ART success is still unknown.

In 2003, Johansson et al. studied the DNA content of blastomeres/fragments using donated excess embryos. They included grade 3 embryos, which were defined as $>20 \%$ but $<50 \%$ fragments and/or blastomeres/cells of all sizes and/or heavily granulated cytoplasm or vacuolation. According to this study, the blastomere size should be over $45 \mu \mathrm{m}$ in the second day embryo and over $40 \mu \mathrm{m}$ in the third day embryo in order to be called a blastomere. They concluded that cells smaller than this were always anucleated and should be considered fragments [14]. This suggests a relationship between the increase in blastomere diameter and impaired development of the embryo. It is possible that this situation will affect pregnancy rates in IVF.

According to the results of the current study published by Gardner et al., low, medium, or high viability classification determined by morphometric evaluation of the embryo is important to the implantation of the embryo and clinical pregnancy rates. Ideal day 3 embryos with high viability are described as the ones with mononucleated blastomeres, equal cell size, $<20 \%$ fragmentation, and at least 4 blastomeres. Thus, ideal embryos are related to higher implantation, pregnancy, and birth rates [15]. Our study is the first to measure MBD and relate the cutoff value to the clinical pregnancy rate. The researchers concluded that bio- and/or morphokinetic markers are important in the selection of the embryo for transfer, among which morphological data play a key role [15].

Alpha Scientists in Reproductive Medicine and the ESHRE Special Interest Group of Embryology organized a meeting in 2010 in Istanbul. At this meeting, oocyte, zygote, and embryo grading criteria were evaluated in the IVF laboratory, and a consensus was reached [6]. According to this consensus, the embryo is expected to have 4 cells on the second day and 8 cells on the third day, depending on the time of ICSI or IVF. Embryo fragmentation was evaluated as mild when below $10 \%$, moderate when it was $10-25 \%$, and severe when it was above $25 \%$. If the appearance of multinucleation is detected, the implantation potential of the embryo will be low, the rate of chromosomal anomalies will be high, and the rate of spontaneous abortion will be high $[6,15]$. Criteria such as blastomere size evaluation, cytoplasmic granularity scoring, membrane appearance scoring, and cytoplasmic vacuole presence grading in the second- or third-day embryo are parameters that can be used in terms of morphometric evaluation. These results were obtained from large series and data from many centers. However, MBD was not defined as a morphometric feature, as in other studies.

According to the results of the study on cases with another single embryo transfer, the effects of parameters such as blastomere volume index, blastomere symmetry index, and mean ovality on the ongoing pregnancy rate were investigated. The third day embryo was evaluated in this context [16]. It was concluded that these parameters, which were established with the help of a computer and determined by special formulas, were not useful in terms of embryo selection criteria [16].

Another question is how important morphometric evaluation before embryo transfer might be or whether multiple evaluations will be required. In a systematic review, Kaser and Racowsky investigated the selection of embryos with a time-lapse monitoring system [17]. In this review, the effects of many morphometric parameters (pronuclear dynamics and morphology, first cytokinesis time, nucleus disappearance time after cleavage, time to reach cleavage phases, cleavage cycle time, time to reach morula, time to reach blastocyst phase, etc.) were investigated. Although many studies have reported that embryos that pass the cleavage stage faster have higher implantation potential, it has been concluded that a single morphokinetic parameter cannot be used to evaluate the embryo with the best implantation potential [17].

There are no studies reporting the relationship between blastomere size and pregnancy rates in IVF. However, of course, we estimate that the pregnancy rate will be high in cases with a high blastomere size, up to a certain limit. As the blastomere size increases, the embryo surface area will also increase. The increase in surface area will also increase the area of interaction between the endometrium and the blastomere surface to be implanted. In this way, pregnancy rates may increase, as there will be a large number of endometrial and embryo receptor interactions.

The most important strength of this study is the selection of the embryo with the best implantation potential using the embryo morphometric measurement to be performed on the day of transfer in cases with single embryo transfer. In this way, an embryo with a high pregnancy rate will be transferred. To our best knowledge, MBD has not previously been proposed as a 
predictor for ICSI outcome. When an embryo with an MBD measurement greater than 49.73 is selected for transfer, in addition to obtaining a high clinical pregnancy rate, multiple pregnancies and their maternal fetal complications may be prevented.

There are some limitations to present study. In this study, the embryo morphological assessment was individual, dependent, and subjective, and there were no intermittent measurement values [18]. Another limitation is the small number of cases. In addition, although all MBD measurements were made by a single embryologist, diameter markings were made manually. The whole study was built on measurements of blastomeres from a single photo, which means that we may have at least 2 blastomeres with no clear outline. This could have some subjective consequences. For the most objective evaluations, a computer-based system would be helpful.

\section{Conclusions}

Our results suggest that a simple morphometric measurement may be a tool for fertility clinics to identify embryos with the greatest implantation potential for single embryo transfer, which would increase the chances of successful outcomes and decrease the risk of multiple births. It is suggested that prospective, randomized controlled trials (RCTs) be performed before use in clinical practice.

\section{Abbreviations}

ART: Assisted reproduction technologies; ICSI: Intracytoplasmic sperm injection; FSH: Follicle stimulating hormone; MBD: Mean blastomere diameter; IVF: In vitro fertilization

\section{Acknowledgements}

Authors thank E. Odaci, E. Yulug, M. A. Unsal, C. Kart, and O. Yildirim for support and help for performing this study.

\section{Authors' contributions}

SG thought, planned, analyzed, and conducted the research. ESGG and SAT wrote the article and made the final revisions. The last corrections and adjustments have been made by SG. All authors have read and approved the manuscript.

\section{Funding}

The authors report no funding sources.

\section{Availability of data and materials}

All data and material mentioned in manuscript would be available upon request.

\section{Declarations}

\section{Ethics approval and consent to participate}

The study protocol was approved by Karadeniz Technical University Faculty of Medicine Ethic Council (Date 03/04/2012, Approval number 2012/26).

Written inform consent was obtained from all participants.

\section{Consent for publication}

Not applicable.

\section{Competing interests}

The authors report no conflict of interest.

\section{Author details}

'Departments of Obstetrics and Gynecology, Karadeniz Technical University Faculty of Medicine, Farabi Hospital, 61080 Trabzon, Turkey. ${ }^{2}$ Departments of Obstetrics and Gynecology, Giresun University School of Medicine, Giresun, Turkey.

Received: 2 November 2020 Accepted: 22 March 2021

Published online: 06 April 2021

\section{References}

1. Palermo GD, Cohen J, Alikani M, Adler A, Rosenwaks Z (1995) Development and implementation of intracytoplasmic sperm injection (ICSI). Reprod Fertil Dev 7(2):211-217; discussion 217-218. https://doi.org/10.1071/RD9950211

2. Bonduelle M, Liebaers I, Deketelaere V, Derde MP, Camus M, Devroey P, Van Steirteghem A (2002) Neonatal data on a cohort of 2889 infants born after ICSI (1991-1999) and of 2995 infants born after IVF (1983-1999). Hum Reprod 17(3):671-694. https://doi.org/10.1093/humrep/17.3.671

3. Hourvitz A, Pri-Paz S, Dor J, Seidman DS (2005) Neonatal and obstetric outcome of pregnancies conceived by ICSI or IVF. Reprod BioMed Online 11(4):469-475. https://doi.org/10.1016/S1472-6483(10)61143-1

4. Sakkas D (2013) Novel technologies for selecting the best sperm for in vitro fertilization and intracytoplasmic sperm injection. Fertil Steril 99(4):10231029. https://doi.org/10.1016/j.fertnstert.2012.12.025

5. Pandian Z, Marjoribanks J, Ozturk O, Serour G, Bhattacharya S (2013) Number of embryos for transfer following in vitro fertilisation or intracytoplasmic sperm injection. Cochrane Database Syst Rev 7:CD003416. https://doi.org/10.1002/14651858.CD003416.pub4

6. Alpha Scientists in Reproductive M, Embryology ESIGo (2011) The Istanbul consensus workshop on embryo assessment: proceedings of an expert meeting. Hum Reprod 26(6):1270-1283. https:/doi.org/10.1093/humrep/der037

7. Guven S, Kart C, Unsal MA, Yildirim O, Odaci E, Yulug E (2014) Endometrial injury may increase the clinical pregnancy rate in normoresponders undergoing long agonist protocol ICSI cycles with single embryo transfer. Eur J Obstet Gynecol Reprod Biol 173:58-62. https://doi.org/10.1016/j. ejogrb.2013.11.005

8. Hardarson T, Hanson C, Sjogren A, Lundin K (2001) Human embryos with unevenly sized blastomeres have lower pregnancy and implantation rates: indications for aneuploidy and multinucleation. Hum Reprod 16(2):313-318. https://doi.org/10.1093/humrep/16.2.313

9. Cooper TG, Noonan E, von Eckardstein S, Auger J, Baker HW, Behre HM, Haugen TB, Kruger T, Wang C, Mbizvo MT, Vogelsong KM (2010) World Health Organization reference values for human semen characteristics. Hum Reprod Update 16(3):231-245. https://doi.org/10.1093/humupd/dmp048

10. Lan KC, Huang FJ, Lin YC, Kung FT, Hsieh CH, Huang HW, Tan PH, Chang SY (2003) The predictive value of using a combined Z-score and day 3 embryo morphology score in the assessment of embryo survival on day 5 . Hum Reprod 18(6):1299-1306. https://doi.org/10.1093/humrep/deg239

11. Schieve LA, Peterson HB, Meikle SF, Jeng G, Danel I, Burnett NM, Wilcox LS (1999) Live-birth rates and multiple-birth risk using in vitro fertilization. JAMA 282(19):1832-1838. https://doi.org/10.1001/jama.282.19.1832

12. De Placido G, Wilding M, Strina I, Alviggi E, Alviggi C, Mollo A, Varicchio MT, Tolino A, Schiattarella C, Dale B (2002) High outcome predictability after IVF using a combined score for zygote and embryo morphology and growth rate. Hum Reprod 17(9):2402-2409. https://doi.org/10.1093/humrep/17.9.2402

13. Practice Committee of Society for Assisted Reproductive T, Practice Committee of American Society for Reproductive M (2008) Guidelines on number of embryos transferred. Fertil Steril 90(5 Suppl):S163-S164. https:// doi.org/10.1016/j.fertnstert.2008.08.053

14. Johansson M, Hardarson T, Lundin K (2003) There is a cutoff limit in diameter between a blastomere and a small anucleate fragment. J Assist Reprod Genet 20(8):309-313. https://doi.org/10.1023/A:1024805407058

15. Gardner DK, Balaban B (2016) Assessment of human embryo development using morphological criteria in an era of time-lapse, algorithms and 'OMICS': is looking good still important? Mol Hum Reprod 22(10):704-718. https:// doi.org/10.1093/molehr/gaw057

16. Vergouw CG, Al Nofal M, Kostelijk EH, Rooth $H$, Hompes PG, Schats R, Lambalk CB (2013) The association of the blastomere volume index (BVI), the blastomere symmetry index (BSI) and the mean ovality (MO) with 
ongoing implantation after single embryo transfer. J Assist Reprod Genet 30(4):587-592. https://doi.org/10.1007/s10815-013-9970-8

17. Kaser DJ, Racowsky C (2014) Clinical outcomes following selection of human preimplantation embryos with time-lapse monitoring: a systematic review. Hum Reprod Update 20(5):617-631. https://doi.org/10.1093/ humupd/dmu023

18. Sjoblom P, Menezes J, Cummins L, Mathiyalagan B, Costello MF (2006) Prediction of embryo developmental potential and pregnancy based on early stage morphological characteristics. Fertil Steril 86(4):848-861. https:// doi.org/10.1016/j.fertnstert.2006.03.040

\section{Publisher's Note}

Springer Nature remains neutral with regard to jurisdictional claims in published maps and institutional affiliations.

\section{Submit your manuscript to a SpringerOpen ${ }^{\circ}$ journal and benefit from:}

- Convenient online submission

- Rigorous peer review

- Open access: articles freely available online

High visibility within the field

- Retaining the copyright to your article

Submit your next manuscript at $\boldsymbol{\nabla}$ springeropen.com 